\begin{tabular}{|r|l|}
\hline \multicolumn{2}{|c|}{ Statistica Sinica Preprint No: SS-2020-0520 } \\
\hline Title & $\begin{array}{l}\text { The Identifiability of Copula Models for Dependent } \\
\text { Competing Risks Data With Exponentially Distributed } \\
\text { Margins }\end{array}$ \\
\hline Manuscript ID & SS-2020-0520 \\
\hline URL & http://www.stat.sinica.edu.tw/statistica// \\
\hline DOI & $10.5705 /$ ss.202020.0520 \\
\hline Complete List of Authors & Antai Wang \\
\hline Corresponding Author & Antai Wang \\
\hline E-mail & aw224@njit.edu \\
\hline Notice: Accepted version subject to English editing. \\
\hline
\end{tabular}




\title{
The identifiability of copula models for dependent competing risks data with

\author{
exponentially distributed margins
}

\author{
Antai Wang \\ Department of Mathematical Sciences \\ New Jersey Institute of Technology \\ Newark, NJ, USA
}

Corresponding author: Antai Wang, Ph.D., Department of Mathematical Sciences, New Jersey Institute of Technology, Newark, NJ, USA Email: aw224@njit.edu. 


\begin{abstract}
In this paper, we prove the identifiability property of Archimedean copula models for dependent competing risks data when at least one of the failure times is exponentially distributed. With this property, it becomes possible to quantify the dependence between competing events based on exponentially distributed dependent censored data. We demonstrate our estimation procedure using simulation studies and illustrate the estimation procedure by applying it to a survival data.

Some key words: Archimedean Copula Models, Copula Graphic Estimator, Identifiability of Competing Risks Data
\end{abstract}

\title{
1. Introduction
}

The identifiability of competing risks model has been a challenging topic in statistical research. Suppose that $\mathrm{T}$ is a time to an event and $C$ is a time to a competing event so that one can only observe $(\min \{T, C\}, I(T<$ $C)$ ). How to evaluate the true association between $T$ and $C$ is an important research issue. Tsiatis (1975) has proved the nonidentifiability of competing risks model for this type of data without any model or covariate information. Wang (2012) has proved the nonidentifiability of Archimedean copula models 
based on dependent censored data. Heckman and Honoré (1989) have proposed a set of conditions to make a competing risks model identifiable with additional covariate information. Wang, Chandra, Xu and Sun (2015) established a set of simpler conditions to make an Archimedean copula model (a special class of competing risks model) identifiable with covariate information.

In survival data analysis, survival times are often assumed to be exponentially distributed. We are interested in finding models that are identifiable when time to an event is exponentially distributed and subject to dependent censoring. It turns out that the Archimedean copula model assumption is good enough to make the corresponding model identifiable for dependent censored data when at least one of the failure times is exponentially distributed (as will be proved in this paper).

Another motivation of this research is related with the estimation of survival functions. The Kaplan-Meier estimator has become the major tool in survival analysis since the estimator was first introduced by Kaplan and Meier (1958). It is a consistent estimator of the survival function of a failure time $T$ when $T$ is subject to independent right censoring with a censoring time $C$. The available data we have is $\left(X_{i}, \delta_{i}\right)=\left(\min \left\{T_{i}, C_{i}\right\}, I\left(T_{i}<C_{i}\right)\right)$ for $i=1,2, \ldots, n$, where $T$ and $C$ are assumed to be independent and the Kaplan-Meier estimator is defined as:

$$
\hat{S}_{T}(t)=\Pi_{X_{i}<t}\left(1-\frac{d_{i}}{n_{i}}\right)
$$


where $d_{i}$ and $n_{i}$ are defined as the number of failure at time $X_{i}$ and the number "at risk" just prior to time $X_{i}$ respectively. Then one can show

$$
\hat{S}_{T}(t) \rightarrow S_{T}(t)=\operatorname{Pr}(T>t)
$$

uniformly when $n \rightarrow \infty$ (see Fleming and Harrington 1991). However, when $T$ and $C$ are dependent, the Kaplan-Meier estimator can no longer be applied because it is a biased estimator of survival function of $T$. Therefore, it is of great importance and research interest to model the dependence structure between $T$ and $C$ and quantify the true dependence between them effectively. Once the dependence level is determined, the marginal survival functions can be consistently estimated using the copula graphic estimator proposed by Zheng and Klein (1996) and Rivest and Wells (2001) or the estimator proposed by Wang (2014).

Our paper is organized in the following way: we present our main results about the identifiability in section 2 . In section 3 , we propose a new approach to estimating the dependence parameter in Archimedean copula models based on the property we have proved in section 2. Simulation studies have demonstrated that the proposed method works well in section 4 . We end our paper with some discussion in section 5 .

\section{Main Results}

A bivariate random vector $(T, C)$ follows an Archimedean copula if the 
joint survival function of $(T, C)$ can be expressed as:

$$
S(t, c)=\psi_{\theta}\left\{\psi_{\theta}^{-1}\left[S_{1}(t)\right]+\psi_{\theta}^{-1}\left[S_{2}(c)\right]\right\}
$$

where $S_{1}$ and $S_{2}$ are marginal survival functions of $T$ and $C$ respectively, $\psi_{\theta}$ is defined on $[0, \infty]$ so that $\psi_{\theta}(0)=1, \psi_{\theta}^{\prime}(s)<0$ and $\psi_{\theta}^{\prime \prime}(s)>0$. $\psi_{\theta}^{-1}$ is the inverse function of $\psi_{\theta}$, defined as a copula generator (see Nelsen 2007) and $\theta$ is the unknown parameter.

The first Archimedean copula model was proposed by Clayton (1978). For this model, $\psi_{\alpha}(s)=(1+s)^{-1 / \alpha}$ which leads to the bivariate survivor function

$$
S(t, c)=\left\{\frac{1}{S_{1}(t)^{-\alpha}+S_{2}(c)^{-\alpha}-1}\right\}^{1 / \alpha}
$$

for $\alpha>0$. Another important frailty model, the Hougaard model (Hougaard 1986), has $\psi_{\beta}(s)=\exp \left(-s^{\beta}\right)$. Its bivariate survivor function is

$$
S(t, c)=\exp \left(-\left[\left\{-\log \left(S_{1}(t)\right)\right\}^{1 / \beta}+\left\{-\log \left(S_{2}(c)\right)\right\}^{1 / \beta}\right]^{\beta}\right)
$$

for $\beta \in(0,1)$. Besides the Clayton model and the Hougaard model, some well-known models such as the Frank model (Genest 1987) and the Log-copula model belong to this family.

For a dependent censored data $(X=\min \{T, C\}, \delta=I(T<C))$ where $(T, C)$ can be modelled by an Archimedean copula, we can prove the following result:

Theorem 1 . For a dependent censored data $(X=\min \{T, C\}, \delta=I(T<$ $C)$ ), assume that the distribution of $(T, C)$ can be modeled by an Archimedean 
copula with the copula generator $\psi_{\theta}$ ( $\theta$ is the dependence parameter):

$$
S(t, c)=\psi_{\theta}\left[\psi_{\theta}^{-1}\left(S_{1}(t)\right)+\psi_{\theta}^{-1}\left(S_{2}(c)\right)\right]
$$

Under the assumption that $\psi_{\theta_{1}}^{-1 \prime} / \psi_{\theta_{2}}^{-1 \prime}$ is a strictly increasing function for $\theta_{1}<$ $\theta_{2}$, there is a unique Archimedean copula model such that the distribution of $T$ (or $C$ ) is exponential on $[0, \infty)$.

Proof: suppose there are two marginal survival functions $S_{1}^{(1)}$ and $S_{1}^{(2)}$ of $T$ corresponding to different dependence levels $\theta_{1}$ and $\theta_{2}$ such that both of them are exponential distributions with the parameter $\lambda_{1}$ and $\lambda_{2}$ respectively, so that:

$$
S_{1}^{(1)}(t)=\exp \left(-\lambda_{1} t\right) \quad S_{1}^{(2)}(t)=\exp \left(-\lambda_{2} t\right)
$$

Then we have two Archimedean copula models with the copula generators $\psi_{\theta_{1}}$ and $\psi_{\theta_{2}}$ respectively such that

$$
S^{(1)}(t, c)=\psi_{\theta_{1}}\left[\psi_{\theta_{1}}^{-1}\left(S_{1}^{(1)}(t)\right)+\psi_{\theta_{1}}^{-1}\left(S_{2}^{(1)}(c)\right)\right]
$$

and

$$
S^{(2)}(t, c)=\psi_{\theta_{2}}\left[\psi_{\theta_{2}}^{-1}\left(S_{1}^{(2)}(t)\right)+\psi_{\theta_{2}}^{-1}\left(S_{2}^{(2)}(c)\right)\right]
$$

and the distributions of $(X, \delta)$ corresponding to both models are the same. Without loss of generality, we assume that $\theta_{1}<\theta_{2}$.

Because the distributions of $(X, \delta)$ are the same for both models, by Theorem 2 of Wang et al (2015), the following equality must hold:

$$
S_{1}^{(2)}(t)=\psi_{\theta_{2}}\left[\int_{0}^{t} \frac{\psi_{\theta_{2}}^{-1 \prime}\{\pi(u)\}}{\psi_{\theta_{1}}^{-1 \prime}\{\pi(u)\}} d \psi_{\theta_{1}}^{-1}\left(S_{1}^{(1)}(u)\right)\right]
$$


Taking derivative with respect to $t$ on both sides we get:

$$
\begin{gathered}
S_{1}^{(2) \prime}(t)=\psi_{\theta_{2}}^{\prime}\left[\int_{0}^{t} \frac{\psi_{\theta_{2}}^{-1 \prime}\{\pi(u)\}}{\psi_{\theta_{1}}^{-1 \prime}\{\pi(u)\}} d \psi_{\theta_{1}}^{-1}\left(S_{1}^{(1)}(u)\right)\right] \frac{\psi_{\theta_{2}}^{-1 \prime}\{\pi(t)\}}{\psi_{\theta_{1}}^{-1 \prime}\{\pi(t)\}} \psi_{\theta_{1}}^{-1 \prime}\left(S_{1}^{(1)}(t)\right) S_{1}^{(1) \prime}(t) \\
=\frac{\psi_{\theta_{2}}^{-1 \prime}\{\pi(t)\}}{\psi_{\theta_{1}}^{-1 \prime}\{\pi(t)\}} \frac{\psi_{\theta_{1}}^{-1 \prime}\left(S_{1}^{(1)}(t)\right)}{\psi_{\theta_{2}}^{-1 \prime}\left(S_{1}^{(2)}(t)\right)} S_{1}^{(1) \prime}(t) .
\end{gathered}
$$

From which we have:

$$
\frac{\psi_{\theta_{1}}^{-1 \prime}\{\pi(t)\}}{\psi_{\theta_{2}}^{-1 \prime}\{\pi(t)\}}=\frac{\psi_{\theta_{1}}^{-1 \prime}\left(S_{1}^{(1)}(t)\right)}{\psi_{\theta_{2}}^{-1 \prime}\left(S_{1}^{(2)}(t)\right)} \times \frac{S_{1}^{(1) \prime}(t)}{S_{1}^{(2) \prime}(t)}
$$

Suppose $S_{1}^{(1)}(t)=S_{1}^{(2)}(t)$, then $S_{1}^{(1) \prime}(t)=S_{1}^{(2) \prime}(t)$. From above equality we must have:

$$
\frac{\psi_{\theta_{1}}^{-1 \prime}\{\pi(t)\}}{\psi_{\theta_{2}}^{-1 \prime}\{\pi(t)\}}=\frac{\psi_{\theta_{1}}^{-1 \prime}\left(S_{1}^{(1)}(t)\right)}{\psi_{\theta_{2}}^{-1 \prime}\left(S_{1}^{(2)}(t)\right)} \times \frac{S_{1}^{(1) \prime}(t)}{S_{1}^{(2) \prime}(t)}=\frac{\psi_{\theta_{1}}^{-1 \prime}\left(S_{1}^{(1)}(t)\right)}{\psi_{\theta_{2}}^{-1 \prime}\left(S_{1}^{(1)}(t)\right)}
$$

which is impossible to hold because we know

$$
\pi(t)=P(T>t, C>t)=S(t, t)=\psi_{\theta}\left[\psi_{\theta}^{-1}\left(S_{1}(t)\right)+\psi_{\theta}^{-1}\left(S_{2}(t)\right)\right]
$$

where $\theta=\theta_{1}$ or $\theta_{2}$ because $\pi(t)=P(T>t, C>t)=S(t, t)$ is the same for both models. It is obvious that $\pi(t)=S(t, t)$ is generally smaller than the probability of $P(T>t)=S_{1}(t)$. By the condition that $\psi_{\theta_{1}}^{-1 \prime} / \psi_{\theta_{2}}^{-1 \prime}$ is a strictly increasing function when $\theta_{1}<\theta_{2}$, we must have

$$
\frac{\psi_{\theta_{1}}^{-1 \prime}\{\pi(t)\}}{\psi_{\theta_{2}}^{-1 \prime}\{\pi(t)\}}<\frac{\psi_{\theta_{1}}^{-1 \prime}\left(S_{1}^{(1)}(t)\right)}{\psi_{\theta_{2}}^{-1 \prime}\left(S_{1}^{(1)}(t)\right)}
$$

and this leads to an contradiction. Therefore, we only need to consider the situation that $S_{1}^{(1)}(t) \neq S_{1}^{(2)}(t)$. 
Now consider the stochastic ordering of $S_{1}^{(1)}(t)$ and $S_{1}^{(2)}(t)$. Under the assumption that $\psi_{\theta_{1}}^{-1 \prime} / \psi_{\theta_{2}}^{-1 \prime}$ is an increasing function when $\theta_{1}<\theta_{2}$, applying Proposition 2 in Rivest and Wells 2001, we can conclude

$$
S_{1}^{(2)}(t) \leq S_{1}^{(1)}(t)
$$

so that $\lambda_{1}<\lambda_{2}$ (because we just proved that $S_{1}^{(1)}(t) \neq S_{1}^{(2)}(t)$ ). Hence

$$
\frac{S_{1}^{(1) \prime}(t)}{S_{1}^{(2) \prime}(t)}=\frac{\lambda_{1} \exp \left(-\lambda_{1} t\right)}{\lambda_{2} \exp \left(-\lambda_{2} t\right)}
$$

Now consider the equality:

$$
\frac{\psi_{\theta_{1}}^{-1 \prime}\{\pi(t)\}}{\psi_{\theta_{2}}^{-1 \prime}\{\pi(t)\}}=\frac{\psi_{\theta_{1}}^{-1 \prime}\left(S_{1}^{(1)}(t)\right)}{\psi_{\theta_{2}}^{-1 \prime}\left(S_{1}^{(2)}(t)\right)} \times \frac{S_{1}^{(1) \prime}(t)}{S_{1}^{(2) \prime}(t)}=\frac{\psi_{\theta_{1}}^{-1 \prime}\left(S_{1}^{(1)}(t)\right)}{\psi_{\theta_{2}}^{-1 \prime}\left(S_{1}^{(2)}(t)\right)} \frac{\lambda_{1} \exp \left(-\lambda_{1} t\right)}{\lambda_{2} \exp \left(-\lambda_{2} t\right)}
$$

Letting $t \rightarrow 0(t \geq 0)$ on both sides, we can see that the limit of the left hand side equals to

$$
\lim _{t \rightarrow 0} \frac{\psi_{\theta_{1}}^{-1 \prime}\{\pi(t)\}}{\psi_{\theta_{2}}^{-1 \prime}\{\pi(t)\}}=\frac{\psi_{\theta_{1}}^{-1 \prime}\{\pi(0)\}}{\psi_{\theta_{2}}^{-1 \prime}\{\pi(0)\}}=\frac{\psi_{\theta_{1}}^{-1 \prime}\{1\}}{\psi_{\theta_{2}}^{-1 \prime}\{1\}}
$$

while the limit of the right hand side equals to

$$
\frac{\psi_{\theta_{1}}^{-1 \prime}\left(S_{1}^{(1)}(0)\right)}{\psi_{\theta_{2}}^{-1 \prime}\left(S_{1}^{(2)}(0)\right)} \times \frac{\lambda_{1}}{\lambda_{2}}=\frac{\psi_{\theta_{1}}^{-1 \prime}\{1\}}{\psi_{\theta_{2}}^{-1 \prime}\{1\}} \frac{\lambda_{1}}{\lambda_{2}} \neq \frac{\psi_{\theta_{1}}^{-1 \prime}\{1\}}{\psi_{\theta_{2}}^{-1 \prime}\{1\}}
$$

because $\psi_{\theta_{1}}^{-1 /}\{1\}$ and $\psi_{\theta_{2}}^{-1 \prime}\{1\}$ are all well defined finite quantities for

Archimedean copula models and $\lambda_{1}<\lambda_{2}$, the equality can't hold on a set with probability measure greater than zero. Contradiction! Therefore we can conclude that there is a unique Archimedean copula model such that the marginal distribution of $T$ is exponential on $[0, \infty)$. 
Remark: the condition $\psi_{\theta_{1}}^{-1 \prime} / \psi_{\theta_{2}}^{-1 \prime}$ being an increasing function for $\theta_{1}<\theta_{2}$ is satisfied for most one parameter families of Archimedean copulas, such as the Frank model or the Clayton model. This fact has been pointed out by Rivest and Wells (2001).

One of the reviewers of this paper raised following question regarding our paper: "the identifiability issue has already been dealt with using copulas in previous studies (e.g. Carrierè 1995 and Escarela G, Carrierè, J 2002). What is different with your methods?" Our answer is that in these two papers, the authors have assumed that both the crude survival functions and the copula function are known to us to identify the marginal survival functions. In this paper, we have only assumed that the joint copula function is Archimedean with the corresponding dependence parameter unspecified, then under the weak assumption that one of the marginal survival function is exponential, the dependence can be uniquely determined based on $(X, \delta)$ and the identifiability of marginal survival functions can be established.

\section{Dependence parameter estimation}

Using Theorem 1 we just proved, we know that our model is identifiable based upon the exponential assumption of the marginal distributions of $T$. Now we propose a new parameter estimator for dependent censored data $\left(X_{i}, \delta_{i}\right)=\left(\min \left\{T_{i}, C_{i}\right\}, I\left(T_{i}<C_{i}\right)\right)$ under above model/distribution assumptions, that is, the joint copula of $(T, C)$ is an Archimedean copula and the 
marginal distribution of $T$ is an exponential distribution.

Define $X_{i}=\min \left\{T_{i}, C_{i}\right\}, \delta_{i}=I\left(T_{i}<C_{i}\right), N_{i}(y)=I\left(X_{i}<y, \delta_{i}=1\right)$, $Y_{i}(y)=I\left(T_{i} \geq y, C_{i} \geq y\right), \bar{N}(y)=\sum_{i=1}^{n} N_{i}(y)$ and $\bar{Y}(y)=\sum_{i=1}^{n} Y_{i}(y)$. According to Fleming \& Harrington (1990) and Rivest and Wells (2001),

$$
M_{i}(t)=N_{i}(t)-\int_{0}^{t} Y_{i}(s) \lambda^{\sharp}(s) d s
$$

and

$$
\bar{M}(t)=\bar{N}(t)-\int_{0}^{t} \bar{Y}(s) \lambda^{\sharp}(s) d s
$$

are martingales with respect to the $\sigma$ field $\mathcal{F}_{t}^{i}=\sigma\left\{I\left(X_{i} \leq t, \delta_{i}=1\right), I\left(X_{i} \leq\right.\right.$ $\left.\left.t, \delta_{i}=0\right)\right\}$ and $\mathcal{F}_{t}=\bigvee_{i=1}^{n} \mathcal{F}_{t}^{i}$ respectively and $\lambda^{\sharp}(s)$ is defined as the crude hazard function of $T$ and we denote the corresponding crude cumulative function and the survival function by $\Lambda^{\sharp}(s)$ and $S^{\sharp}(s)$ respectively.

Using Theorem 1 in Wang (2014) or the formula (8) in Rivest and Wells (2001), we can express the marginal survival function of $T$ as:

$$
S_{1}(t)=\psi_{\theta}\left[\int_{0}^{t} \psi_{\theta}^{-1 \prime}(\pi(u)) \pi(u) d \ln \left(S_{1}^{\sharp}(u)\right)\right]
$$

which can be estimated by (see Wang 2014):

$$
\hat{S}_{1}(t)=\psi_{\theta}\left\{-\sum_{X_{i} \leq t, \delta_{i}=1} \psi_{\theta}^{-1 \prime}\left\{\hat{\pi}\left(X_{i}\right)\right\} \hat{\pi}\left(X_{i}\right) \frac{d \bar{N}\left(X_{i}\right)}{\bar{Y}\left(X_{i}\right)}\right\},
$$

where $\psi_{\theta}^{-1 \prime}(u)$ is the derivative of $\psi_{\theta}^{-1}$ with respect to $u$ and $\pi\left(X_{i}\right)=\operatorname{Pr}(X>$ $X_{i}$ ) where $\hat{\pi}\left(X_{i}\right)$ is the empirical survival function of $\pi(X)$ evaluated at $X_{i}$.

The dependence parameter $\theta$ can be estimated in the following way: because $S_{1}(t)$ is assumed to be the survival function of an exponential distribu- 
tion, $S_{1}(t)=\exp (-\lambda t)$ for some $\lambda$ value. The cumulative hazard function of $T$ is:

$$
H(t)=-\log \left(S_{1}(t)\right)=\lambda t
$$

and $H(t) / t=\lambda=1 / \mu$ where $\mu$ is the mean value of $T$. Given a dependent censored data, a natural estimator of $\mu$ can be established as:

$$
\hat{\mu}=\left[\frac{1}{n} \sum_{i=1}^{n} \hat{H}_{1}\left(X_{i}\right) / X_{i}\right]^{-1}=\left[\frac{1}{n} \sum_{i=1}^{n}-\log \hat{S}_{1}\left(X_{i}\right) / X_{i}\right]^{-1}
$$

where $\hat{S}_{1}$ is the copula graphic estimator of $S_{1}$ (see Rivest and Wells 2001) or the Wang estimator of $S_{1}$ (see Wang 2014). The survival function of the corresponding exponential distribution can therefore be alternatively estimated by $\exp \left(-X_{i} / \hat{\mu}\right)$.

The dependence parameter value can then be determined as:

$$
\hat{\theta}_{n}=\operatorname{argmin}_{\theta \in \Theta} Q(\theta)=\operatorname{argmin}_{\theta \in \Theta} \sum_{i=1}^{n}\left\{\hat{S}_{1}\left(X_{i}\right)-\exp \left(-\frac{X_{i}}{\hat{\mu}}\right)\right\}^{2} .
$$

Assuming the differentiability of $\hat{S}_{1}$ with respect to unknown parameter $\theta$, the corresponding estimating equation can be written as

$$
\frac{d Q}{d \theta}=2 \sum_{i=1}^{n}\left\{\hat{S}_{1}\left(X_{i}\right)-\exp \left(-\frac{X_{i}}{\hat{\mu}}\right)\right\}\left\{\frac{d \hat{S}_{1}\left(X_{i}\right)}{d \theta}-\exp \left(-\frac{X_{i}}{\hat{\mu}}\right) \frac{X_{i}}{\hat{\mu}^{2}} \frac{d \hat{\mu}}{d \theta}\right\}=0
$$

Asymptotic normality of our parameter estimator follows based on the corresponding Taylor expansion of our estimating equation under necessary regularity conditions.

Theorem 2 . Under necessary regularity conditions, $\hat{\theta}_{n}$ is consistent and $\sqrt{n}\left(\hat{\theta}_{n}-\theta_{0}\right)$ is asymptotically normal with mean zero and a finite variance $\sigma^{2}$. 
For proof details of Theorem 2, see Appendix. The variance formula is quite complicated and we recommend to use bootstrap variance estimator to estimate the variance.

\section{Simulation studies}

We have conducted some simulation studies to demonstrate our parameter estimation procedure. We consider weak to strong dependence levels with Kendall's $\tau$ values equal to $0.3,0.5$, and 0.7 for the Clayton copula model when the sample sizes $n=500, n=1500$ and $n=3000$ respectively. We generate $(T, C)$ from a Clayton copula model with unit exponential marginal distributions and estimate the dependence parameter $\alpha=2 \tau /(1-\tau)$ using our proposed method described in Section 3. Based on any simulated sample, we get a bootstrap sample using the resampling approach. We estimated the parameter using our proposed method for bootstrap samples B times to get a bootstrap variance estimate using bootstrap estimates of the parameter. We then take the average of the bootstrap variance estimate which should be approximate the sample variance of our proposed parameter estimates. For each simulated sample, we can get the confidence interval for our parameter using the bootstrap variance estimate.

The simulation results are listed in Table 1 . In Table $1, \hat{\alpha}_{1}$ represents our proposed estimate of $\alpha, \hat{\alpha}_{2}$ represents the MLE estimate of $\alpha$ assuming that the joint survival can be modelled by the Clayton model and marginal distributions 
are all exponentially distributions. $\hat{\alpha}_{3}$ represents the estimate of the parameter $\alpha$ by minimizing the sum of distances between the copula-graphic estimates of $S_{T}$ and the survival function estimates of $T$ and also distances between the copula-graphic estimates of $S_{C}$ and the survival function estimates of $C$ assuming that the joint survival can be modelled by the Clayton model and marginal distributions are all exponentially distributions.

From Table 1, we can see that our proposed estimation approach works well for different levels of dependence under the Clayton model assumption. When the sample sizes increase from $n=500$ to $n=3000$, the proposed parameter estimates tend to be less biased and in both scenarios, the bootstrap variance estimators work reasonably well because they are close to the sample variances of the proposed parameter estimates. In general, these results demonstrate the identifiability of our models when the marginal distributions of $T$ is exponential. In addition, we also see that the $\operatorname{MLE}\left(\hat{\alpha}_{2}\right)$ and $\hat{\alpha}_{3}$ tend to be more efficient than proposed parameter estimates by comparing sample variances and also less biased (not surprisingly, MLE is the most efficient estimate). This is understandable because we have more information about the data (here we assume both marginal distributions are exponential rather than that only one margin is exponentially distributed, the assumptions under which our proposed estimate $\hat{\alpha}_{1}$ is developed). 
Table 1: The Clayton model: performance of our parameter estimates based on 1000 repetitions with sample sizes $N=500, N=1500$ and $N=3000$ respectively. $\overline{\hat{\alpha}_{1}}, \overline{\hat{\alpha}_{2}}$ and $\overline{\hat{\alpha}_{3}}$ are mean values of $\hat{\alpha}_{1}, \hat{\alpha}_{2}$ and $\hat{\alpha}_{3}$ respectively. $\widehat{\operatorname{var}}\left(\hat{\alpha}_{1}\right), \widehat{\operatorname{var}}\left(\hat{\alpha}_{2}\right)$ and $\widehat{\operatorname{var}}\left(\hat{\alpha}_{3}\right)$ represent the sample variance of $\hat{\alpha}_{1}, \hat{\alpha}_{2}$ and $\hat{\alpha}_{3}$ respectively. $\overline{\widehat{\operatorname{bvar}(\hat{\alpha})_{1}}}$ represents the mean of the bootstrap variances of the proposed parameter estimate $\hat{\alpha}_{1}$.

\begin{tabular}{cccccccc}
$\alpha$ & $\overline{\hat{\alpha}_{1}}$ & $\overline{\hat{\alpha}_{2}}$ & $\overline{\hat{\alpha}_{3}}$ & $\widehat{\operatorname{var}}\left(\hat{\alpha}_{1}\right)$ & $\overline{\operatorname{bvar}\left(\hat{\alpha}_{1}\right)}$ & $\widehat{\operatorname{var}}\left(\hat{\alpha}_{2}\right)$ & $\widehat{\operatorname{var}}\left(\hat{\alpha}_{3}\right)$ \\
0.86 & 2.11 & 0.81 & 1.81 & 5.48 & 4.30 & 0.25 & 4.37 \\
2.00 & 3.13 & 2.31 & 2.79 & 6.00 & 7.52 & 6.52 & 2.94 \\
4.67 & 6.72 & 5.93 & 7.16 & 16.96 & 14.60 & 14.63 & 14.57 \\
& & & & & & & \\
0.86 & 1.12 & 0.83 & 0.98 & 1.54 & 2.42 & 0.05 & 0.85 \\
2.00 & 2.93 & 2.19 & 3.02 & 6.99 & 4.11 & 0.32 & 1.30 \\
4.67 & 5.26 & 5.08 & 5.52 & 5.31 & 8.57 & 1.71 & 4.73 \\
\hline 4.67 & 5.21 & 4.79 & 5.09 & 4.11 & 4.53 & 0.65 & 1.71
\end{tabular}




\section{An Illustrative Example}

In this section, we illustrate our methodology by analyzing a bone marrow transplantation data described in Klein and Moeschberger (1997). The data set is collected during a study in which 137 patients are followed in their recovery from Leukemia after bone marrow transplantation. We are interested in the disease-free survival time $T$, i.e., the time until a relapse of leukemia. The patients can be censored by two possible events: disease-free death or disease-free and alive at the end of the study. The censoring time $C$ is then defined as the time until the first of these two events happen. Assuming that $T$ is exponentially distributed and applying our estimation method, we find that the association level is $\hat{\alpha}=3.62(\hat{\tau}=0.64)$ with the bootstrap standard error 0.93 under the Clayton model assumption. The 95\% confidence interval for $\alpha$ is $\mathrm{CI}=[3.62-1.96 * 0.93,3.62+1.96 * 0.93]=[1.80,5.44]$ with $0 \notin \mathrm{CI}$. There is enough evidence to conclude that the dependence between $T$ and $C$ is significantly different from zero. This conclusion is consistent with the results given in Lakahal, Rivest and Abdous (2008) and Wang et al (2015). In Lakahal, Rivest and Abdous (2008), the authors explore the relationship between $T$ and the time to death (different from our censoring variable $C$ ) for the same data and found a strong relationship between $T$ and the time to death using a semicompeting risks model.

Based on our parameter estimate, we can estimate the survival function 
Figure 1. Comparison of survival Curves

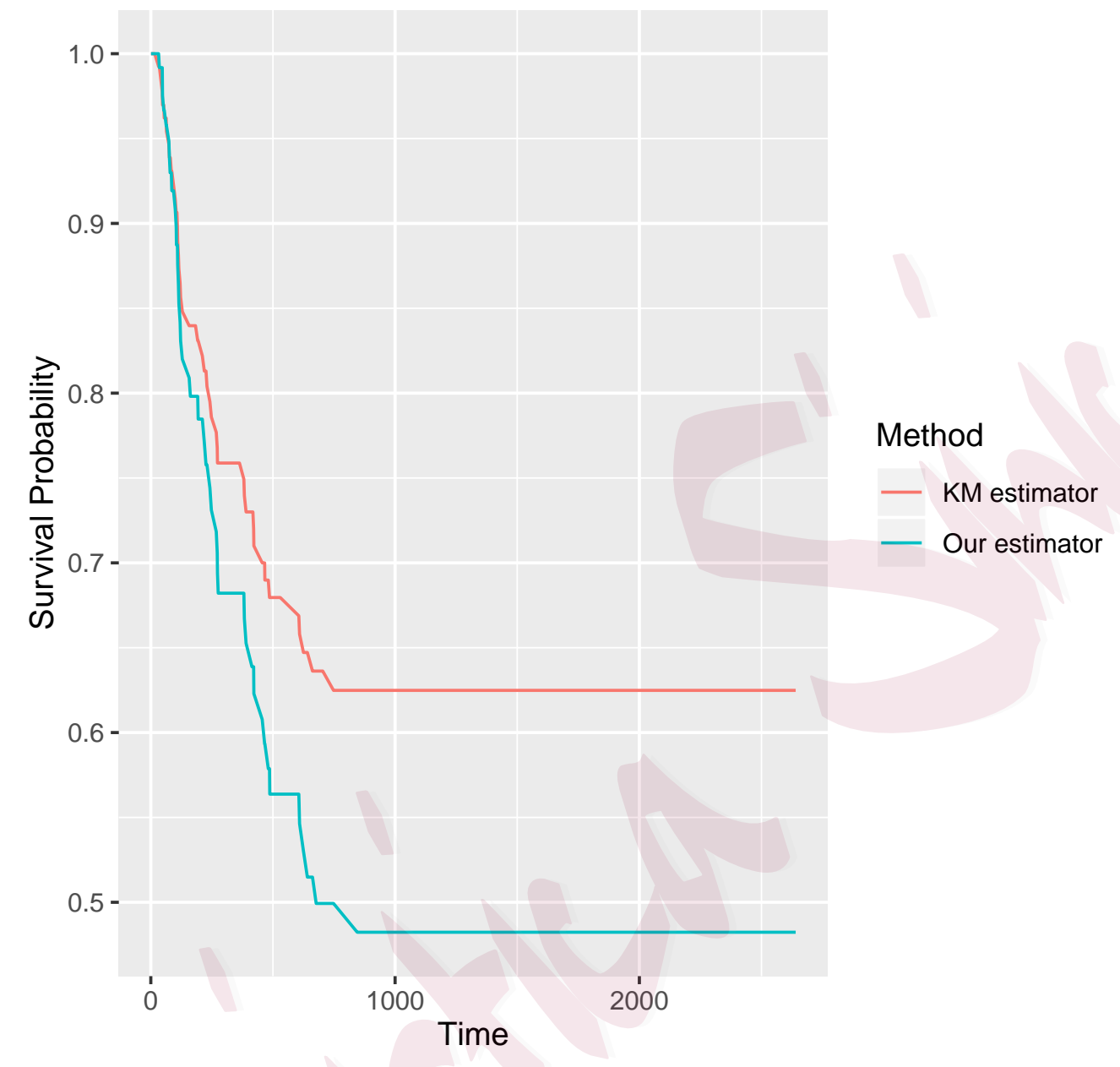

using the copula-graphic estimate proposed by Rivest and Wells (2001) or the Wang estimate proposed in Wang (2014) (these two estimates are equivalent asymptotically as has been shown in Wang 2014). We plot the Wang estimate and the Kaplan-Meier estimate for the same data in the same figure. From Figure 1, we can see that the Kaplan-Meier curve overestimates the survival function when the association is strong. There is a significant difference between two survival function estimates based on the same censored data. 
Therefore it is important to quantify the dependence level accurately to get reasonable survival function estimates.

The advantage of using our approach is that we can directly estimate the association between $T$ and $C$ based on a simple exponential distribution assumption of $T$ which is quite widely used in survival analysis. We don't need to assume a special relationship between $T$ and $C$ (such as $T<C$ in semicompeting risks setting) to make such association estimable (see Lakahal, Rivest and Abdous 2008 and Fine, Jiang and Chappell 2001).

\section{Discussion}

The identifiability of competing risks models has been a long standing problem for quite a while. Because of it, analysis of dependent competing risks data has been a quite challenging task. This research is an attempt to solve this problem by imposing simple copula model and distribution assumptions for dependent censored data.

In this paper, we have proved the identifiability of Archimedean copula models for dependent competing risks data when either failure time $T$ or censoring time $C$ is exponentially distributed. We have also proposed a parameter estimation approach based on our model and distribution assumptions. In survival data analysis, researchers often assume the exponential distributions for time to event data. Therefore, our method should be quite useful for statistical analyses of dependent censored data. Once we obtain consistent estimates of 
the dependence parameter, the association of $\mathrm{T}$ and $\mathrm{C}$ can be tested accordingly, and significance tests can be developed based on the asymptotic theory described in Zeng et al. (2008) for semiparametric transformation models (see theorems 2 and 3 in their paper). The marginal survival functions can be consistently estimated using the copula-graphic estimator (Zheng \& Klein, 1995, Rivest \& Wells, 2001) or the estimator proposed by Wang (2014).

\section{Acknowlegements}

The author would like to thank the Editor and three anonymous reviewers for their encouragement and valuable comments that led to great improvement of this paper.

\section{Appendix}

Proof of consistency of our parameter estimator: by the strong law of large numbers, it is easy to show that

$$
\begin{gathered}
M_{n}(\theta)=\frac{1}{n} \sum_{i=1}^{n}\left[\hat{S}_{1}\left(X_{i}\right)-\exp \left(-X_{i} / \hat{\mu}\right)\right]^{2} \\
\stackrel{p}{\rightarrow} \mathrm{E}\left[S_{1}(X)-\exp (-X / \mu)\right]^{2}=M(\theta)
\end{gathered}
$$

when $n \rightarrow \infty$ based on the following facts: (a) $\hat{S}_{1}\left(X_{i}\right) \stackrel{p}{\rightarrow} S_{1}\left(X_{i}\right)$ uniformly when $n \rightarrow \infty$ by Theorem 1 in Rivest and Wells (2001); (b) $\exp \left(-X_{i} / \hat{\mu}\right) \stackrel{p}{\rightarrow}$ 
$\exp \left(-X_{i} / \mu\right)$ uniformly when $n \rightarrow \infty$ because of (a), the boundedness of corresponding derivatives and the strong law of large numbers.

By Theorem 1 we know there is a unique $\theta=\theta_{0}$ value such that $S_{1}(X)=$ $\exp (-X / \mu)$ and $M(\theta)$ is a continuous function of $\theta$ on a compact set $\Theta$, therefore, we must have

$$
\min _{\theta: d\left(\theta, \theta_{0}\right) \geq \epsilon} M(\theta)>M\left(\theta_{0}\right)=0
$$

$M_{n}(\hat{\theta}) \leq M_{n}(\hat{\theta})+o_{p}(1)$ is trivially satisfied because $\hat{\theta}$ is an M-estimator. Therefore we can conclude that $\hat{\theta} \rightarrow \theta_{0}$ when $n \rightarrow \infty$ by Theorem 5.7 in van der Vaart (2007).

Proof of asymptotic normality of our parameter estimator: using the Taylor expansion at $\theta=\theta_{0}$ (assuming that $\theta_{0}$ is the true parameter), our estimating equation can be written as:

$$
\begin{aligned}
0= & \sum_{i=1}^{n} g_{n}\left(X_{i}, \hat{\theta}\right) \approx \sum_{i=1}^{n} g_{n}\left(X_{i}, \theta_{0}\right) \\
& +\sum_{i=1}^{n} \frac{d g_{n}\left(X_{i}, \theta_{0}\right)}{d \theta}\left(\hat{\theta}-\theta_{0}\right)
\end{aligned}
$$

where $g_{n}\left(X_{i}, \theta_{0}\right)$ is defined as:

$$
\begin{aligned}
g_{n}\left(X_{i}, \theta_{0}\right)= & \left\{\hat{S}_{1}\left(X_{i}\right)-\exp \left(-\frac{X_{i}}{\hat{\mu}}\right)\right\}\left\{\frac{d \hat{S}_{1}\left(X_{i}\right)}{d \theta}-\exp \left(-\frac{X_{i}}{\hat{\mu}}\right) \frac{X_{i}}{\hat{\mu}^{2}} \frac{d \hat{\mu}}{d \theta}\right\} \\
= & \hat{S}_{1}\left(X_{i}\right) \frac{d \hat{S}_{1}\left(X_{i}\right)}{d \theta}-\hat{S}_{1}\left(X_{i}\right) \exp \left(-\frac{X_{i}}{\hat{\mu}}\right) \frac{X_{i}}{\hat{\mu}^{2}} \frac{d \hat{\mu}}{d \theta} \\
& -\exp \left(-\frac{X_{i}}{\hat{\mu}}\right) \frac{d \hat{S}_{1}\left(X_{i}\right)}{d \theta}+\exp \left(-\frac{2 X_{i}}{\hat{\mu}}\right) \frac{X_{i}}{\hat{\mu}^{2}} \frac{d \hat{\mu}}{d \theta}
\end{aligned}
$$


Therefore

$$
\sqrt{n}\left(\hat{\theta}-\theta_{0}\right)=\frac{\frac{\sqrt{n}}{n} \sum_{i=1}^{n} g_{n}\left(X_{i}, \theta_{0}\right)}{\frac{1}{n} \sum_{i=1}^{n} \frac{-d g_{n}\left(X_{i}, \theta_{0}\right)}{d \theta}} .
$$

Noticing the fact that when $\theta=\theta_{0}, S_{1}\left(X_{i}\right)-\exp \left(-X_{i} / \mu\right)=0$ so that

$$
\left\{S_{1}\left(X_{i}\right)-\exp \left(-\frac{X_{i}}{\mu}\right)\right\}\left\{\frac{d S_{1}\left(X_{i}\right)}{d \theta}-\exp \left(-\frac{X_{i}}{\mu}\right) \frac{X_{i}}{\mu^{2}} \frac{d \mu}{d \theta}\right\}=0 .
$$

Therefore,

$$
\sum_{i=1}^{n} g_{n}\left(X_{i}, \theta_{0}\right)=\text { Term } 1+\text { Term } 2+\text { Term } 3+\text { Term } 4
$$

Where

$$
\begin{gathered}
\text { Term } 1=\sum_{i=1}^{n} \hat{S}_{1}\left(X_{i}\right) \frac{d \hat{S}_{1}\left(X_{i}\right)}{d \theta}-S_{1}\left(X_{i}\right) \frac{d S_{1}\left(X_{i}\right)}{d \theta} \\
=\sum_{i=1}^{n} \hat{S}_{1}\left(X_{i}\right) \frac{d \hat{S}_{1}\left(X_{i}\right)}{d \theta}-\hat{S}_{1}\left(X_{i}\right) \frac{d S_{1}\left(X_{i}\right)}{d \theta}+\hat{S}_{1}\left(X_{i}\right) \frac{d S_{1}\left(X_{i}\right)}{d \theta}-S_{1}\left(X_{i}\right) \frac{d S_{1}\left(X_{i}\right)}{d \theta} \\
=\sum_{i=1}^{n} \hat{S}_{1}\left(X_{i}\right)\left\{\frac{d \hat{S}_{1}\left(X_{i}\right)}{d \theta}-\frac{d S_{1}\left(X_{i}\right)}{d \theta}\right\}+\frac{d S_{1}\left(X_{i}\right)}{d \theta}\left\{\hat{S}_{1}\left(X_{i}\right)-S_{1}\left(X_{i}\right)\right\}
\end{gathered}
$$

Here

$$
\hat{S}_{1}(t)=\psi_{\theta}\left\{-\int_{0}^{t} \psi_{\theta}^{-1 \prime}\{\hat{\pi}(u)\} \hat{\pi}(u) \frac{d \bar{N}(u)}{\bar{Y}(u)}\right\}
$$

and

$$
\begin{gathered}
\frac{d \hat{S}_{1}(t)}{d \theta} \\
=\psi_{\theta}^{\prime}\left\{-\int_{0}^{t} \psi_{\theta}^{-1 \prime}\{\hat{\pi}(u)\} \hat{\pi}(u) \frac{d \bar{N}(u)}{\bar{Y}(u)}\right\}\left\{-\int_{0}^{t} \frac{d \psi_{\theta}^{-1 \prime}}{d \theta}\{\hat{\pi}(u)\} \hat{\pi}(u) \frac{d \bar{N}(u)}{\bar{Y}(u)}\right\} \\
=\frac{\left\{-\int_{0}^{t} \frac{d \psi_{\theta}^{-1 \prime}}{d \theta}\{\hat{\pi}(u)\} \hat{\pi}(u) \frac{d \bar{N}(u)}{\bar{Y}(u)}\right\}}{\psi_{\theta}^{-1 \prime}\left[\psi_{\theta}\left[\left\{-\int_{0}^{t} \psi_{\theta}^{-1 \prime}\{\hat{\pi}(u)\} \hat{\pi}(u) \frac{d \bar{N}(u)}{\bar{Y}(u)}\right\}\right]\right]}=\frac{\left\{-\int_{0}^{t} \frac{d \psi_{\theta}^{-1 \prime}}{d \theta}\{\hat{\pi}(u)\} \hat{\pi}(u) \frac{d \bar{N}(u)}{\bar{Y}(u)}\right\}}{\psi_{\theta}^{-1 \prime}\left[\hat{S}_{1}(t)\right]}
\end{gathered}
$$

hence

$$
\frac{d \hat{S}_{1}(t)}{d \theta}-\frac{d S_{1}(t)}{d \theta}
$$




$$
\begin{aligned}
& =\frac{\left\{-\int_{0}^{t} \frac{d \psi_{\theta}^{-1 \prime}}{d \theta}\{\hat{\pi}(u)\} \hat{\pi}(u) \frac{d \bar{N}(u)}{\bar{Y}(u)}\right\}}{\psi_{\theta}^{-1,}\left[\hat{S}_{1}(t)\right]}-\frac{\left\{-\int_{0}^{t} \frac{d \psi_{\theta}^{-1 \prime}}{d \theta}\{\pi(u)\} \pi(u) d \Lambda^{\sharp}(u)\right\}}{\psi_{\theta}^{-1}\left[S_{1}(t)\right]} \\
& =\frac{\left\{-\int_{0}^{t} \frac{d \psi_{\theta}^{-1 \prime}}{d \theta}\{\hat{\pi}(u)\} \hat{\pi}(u) \frac{d \bar{N}(u)}{\bar{Y}(u)}\right\}}{\psi_{\theta}^{-1 \prime}\left[\hat{S}_{1}(t)\right]}-\frac{\left\{-\int_{0}^{t} \frac{d \psi_{\theta}^{-1 \prime}}{d \theta}\{\pi(u)\} \pi(u) d \Lambda^{\sharp}(u)\right\}}{\psi_{\theta}^{-1 \prime}\left[\hat{S}_{1}(t)\right]} \\
& +\frac{\left\{-\int_{0}^{t} \frac{d \psi_{\theta}^{-1 \prime}}{d \theta}\{\pi(u)\} \pi(u) d \Lambda^{\sharp}(u)\right\}}{\psi_{\theta}^{-1 \prime}\left[\hat{S}_{1}(t)\right]}-\frac{\left\{-\int_{0}^{t} \frac{d \psi_{\theta}^{-1 \prime}}{d \theta}\{\pi(u)\} \pi(u) d \Lambda^{\sharp}(u)\right\}}{\psi_{\theta}^{-1}\left[S_{1}(t)\right]} \\
& =\frac{1}{\psi_{\theta}^{-1 \prime}\left[\hat{S}_{1}(t)\right]}\left[-\int_{0}^{t} \frac{d \psi_{\theta}^{-1 \prime}}{d \theta}\{\hat{\pi}(u)\} \hat{\pi}(u) \frac{\Delta \bar{N}(u)}{\bar{Y}(u)}+\int_{0}^{t} \frac{d \psi_{\theta}^{-1 \prime}}{d \theta}\{\pi(u)\} \pi(u) d \Lambda^{\sharp}(u)\right] \\
& +\left\{-\int_{0}^{t} \frac{d \psi_{\theta}^{-1 \prime}}{d \theta}\{\pi(u)\} \pi(u) d \Lambda^{\sharp}(u)\right\}\left[\frac{1}{\psi_{\theta}^{-1 \prime}\left[\hat{S}_{1}(t)\right]}-\frac{1}{\psi_{\theta}^{-1 \prime}\left[S_{1}(t)\right]}\right]
\end{aligned}
$$

Mimicking the arguments given in Rivest and Wells (2001), we can show that

$$
\begin{gathered}
-\int_{0}^{t} \frac{d \psi_{\theta}^{-1 \prime}}{d \theta}\{\hat{\pi}(u)\} \hat{\pi}(u) \frac{d \bar{N}(u)}{\bar{Y}(u)}+\int_{0}^{t} \frac{d \psi_{\theta}^{-1 \prime}}{d \theta}\{\pi(u)\} \pi(u) d \Lambda^{\sharp}(u) \\
=-\frac{1}{n} \int_{0}^{t} \frac{d \psi_{\theta}^{-1 \prime}}{d \theta}\left\{\frac{\bar{Y}}{n}\right\} d \bar{M}(u)+\int_{0}^{t}\left[-\frac{d \psi_{\theta}^{-1 \prime}}{d \theta}\left\{\frac{\bar{Y}}{n}\right\} \frac{\bar{Y}(u)}{n}\right. \\
\left.+\frac{d \psi_{\theta}^{-1 \prime}}{d \theta}\{\pi(u)\} \pi(u)\right] d \Lambda^{\sharp}(u)
\end{gathered}
$$

and also

$$
\begin{gathered}
-\frac{1}{\sqrt{n}} \int_{0}^{t} \frac{d \psi_{\theta}^{-1 \prime}}{d \theta}\left\{\frac{\bar{Y}(u)}{n}\right\} d \bar{M}(u)=-\frac{1}{\sqrt{n}} \int_{0}^{t} \frac{d \psi_{\theta}^{-1 \prime}}{d \theta}\{\pi(u)\} d \bar{M}(u)+o_{p}(1) . \\
-\frac{d \psi_{\theta}^{-1 \prime}}{d \theta}\left\{\frac{\bar{Y}}{n}\right\} \frac{\bar{Y}(u)}{n}+\frac{d \psi_{\theta}^{-1 \prime}}{d \theta}\{\pi(u)\} \pi(u)=\Phi_{1}^{\prime}(\pi(u))\left[\frac{\bar{Y}(u)}{n}-\pi(u)\right]+o_{p}\left(n^{-1 / 2}\right) \\
=\Phi_{1}^{\prime}(\pi(u)) \frac{1}{n} \sum_{i=1}^{n}\left[I\left(X_{i}>u\right)-\pi(u)\right]+o_{p}\left(n^{-1 / 2}\right)
\end{gathered}
$$

where $\Phi_{1}$ is defined as $\Phi_{1}(s)=-s \frac{d \psi_{\theta}^{-1 \prime}}{d \theta}(s)$. Combining these two results, we have

$$
-\int_{0}^{t} \frac{d \psi_{\theta}^{-1 \prime}}{d \theta}\{\hat{\pi}(u)\} \hat{\pi}(u) \frac{d \bar{N}(u)}{\bar{Y}(u)}+\int_{0}^{t} \frac{d \psi_{\theta}^{-1 \prime}}{d \theta}\{\pi(u)\} \pi(u) d \Lambda^{\sharp}(u)
$$




$$
\begin{gathered}
=\frac{1}{\sqrt{n}}\left[-\frac{1}{\sqrt{n}} \int_{0}^{t} \frac{d \psi_{\theta}^{-1 \prime}}{d \theta}\{\pi(u)\} d \bar{M}(u)\right. \\
\left.+\frac{1}{\sqrt{n}} \int_{0}^{t} \sum_{i=1}^{n} \Phi_{1}^{\prime}(\pi(u))\left[I\left(X_{i}>u\right)-\pi(u)\right] d \Lambda^{\sharp}(u)+o_{p}(1)\right]
\end{gathered}
$$

Similarly, we have

$$
\begin{aligned}
&\left\{-\int_{0}^{t} \frac{d \psi_{\theta}^{-1 \prime}}{d \theta}\{\pi(u)\} \pi(u) d \Lambda^{\sharp}(u)\right\}\left[\frac{1}{\psi_{\theta}^{-1 \prime}\left[\hat{S}_{1}(t)\right]}-\frac{1}{\psi_{\theta}^{-1 \prime}\left[S_{1}(t)\right]}\right] \\
&=\left\{-\int_{0}^{t} \frac{d \psi_{\theta}^{-1 \prime}}{d \theta}\{\pi(u)\} \pi(u) d \Lambda^{\sharp}(u)\right\} \frac{\psi_{\theta}^{-1 \prime}\left(S_{1}(t)\right)}{\left[\psi_{\theta}^{-1 \prime}\left(S_{1}(t)\right)\right]^{2}}\left[\hat{S}_{1}(t)-S_{1}(t)\right] \\
&=\left\{-\int_{0}^{t} \frac{d \psi_{\theta}^{-1 \prime}}{d \theta}\{\pi(u)\} \pi(u) d \Lambda^{\sharp}(u)\right\} \frac{\psi_{\theta}^{-1 \prime}\left(S_{1}(t)\right)}{\left[\psi_{\theta}^{-1 \prime}\left(S_{1}(t)\right)\right]^{2}} \frac{1}{\left[\psi_{\theta}^{-1 \prime}\left(S_{1}(t)\right)\right]} \\
& \times\left.\int_{0}^{t} \psi_{\theta}^{-1 \prime}\{\hat{\pi}(u)\} \hat{\pi}(u) \frac{d \bar{N}(u)}{\bar{Y}(u)}+\int_{0}^{t} \psi_{\theta}^{-1 \prime}\{\pi(u)\} \pi(u) d \Lambda^{\sharp}(u)\right\} \\
&=\left\{-\int_{0}^{t} \frac{d \psi_{\theta}^{-1 \prime}}{d \theta}\{\pi(u)\} \pi(u) d \Lambda^{\sharp}(u)\right\} \frac{\psi_{\theta}^{-1 \prime}\left(S_{1}(t)\right)}{\left[\psi_{\theta}^{-1 \prime}\left(S_{1}(t)\right)\right]^{3}} \\
&\left.+\frac{1}{\sqrt{n}} \int_{0}^{t} \sum_{i=1}^{n} \frac{1}{\sqrt{n}}\left[-\frac{1}{\sqrt{n}} \int_{0}^{t} \psi_{\theta}^{-1 \prime}\{\pi(u)\} d \bar{M}^{\prime}(u)\right)\left[I\left(X_{i}>u\right)-\pi(u)\right] d \Lambda^{\sharp}(u)+o_{p}(1)\right] .
\end{aligned}
$$

where $\Phi(s)=-s \psi_{\theta}^{-1 \prime}(s)$. Therefore,

$$
\begin{gathered}
\sqrt{n}\left[\frac{d \hat{S}_{1}\left(X_{i}\right)}{d \theta}-\frac{d S_{1}\left(X_{i}\right)}{d \theta}\right]=\frac{1}{\sqrt{n}} \frac{1}{\psi_{\theta}^{-1 \prime}\left[S_{1}\left(X_{i}\right)\right]} \int_{0}^{X_{i}}-\frac{d \psi_{\theta}^{-1 \prime}}{d \theta}\{\pi(u)\} d \bar{M}(u) \\
+\frac{1}{\sqrt{n}} \frac{1}{\psi_{\theta}^{-1 \prime}\left[S_{1}\left(X_{i}\right)\right]} \int_{0}^{X_{i}} \sum_{j=1}^{n} \Phi_{1}^{\prime}(\pi(u))\left[I\left(X_{j}>u\right)-\pi(u)\right] d \Lambda^{\sharp}(u) \\
+\frac{1}{\sqrt{n}} \sum_{j=1}^{n}\left\{-\int_{0}^{X_{i}} \frac{d \psi_{\theta}^{-1 \prime}}{d \theta}\{\pi(u)\} \pi(u) d \Lambda^{\sharp}(u)\right\} \frac{\psi_{\theta}^{-1 \prime}\left(S_{1}\left(X_{i}\right)\right)}{\left[\psi_{\theta}^{-1 \prime}\left(S_{1}\left(X_{i}\right)\right]^{3}\right.} \\
\times\left[\int_{0}^{X_{i}}-\psi_{\theta}^{-1 \prime}\{\pi(u)\} d M_{j}(u)+\int_{0}^{X_{i}} \Phi^{\prime}(\pi(u))\left[I\left(X_{j}>u\right)-\pi(u)\right] d \Lambda^{\sharp}(u)\right]+o_{p}(1) \\
=\frac{1}{\sqrt{n}} \sum_{j=1}^{n}\left[\frac{1}{\psi_{\theta}^{-1 \prime}\left[S_{1}\left(X_{i}\right)\right]} \int_{0}^{X_{i}}-\psi_{\theta}^{-1 \prime}\{\pi(u)\} d M_{j}(u)\right.
\end{gathered}
$$




$$
\begin{gathered}
\left.+\frac{1}{\psi_{\theta}^{-1 \prime}\left[S_{1}\left(X_{i}\right)\right]} \int_{0}^{X_{i}} \Phi_{1}^{\prime}(\pi(u))\left[I\left(X_{j}>u\right)-\pi(u)\right] d \Lambda^{\sharp}(u)\right] \\
+\frac{1}{\sqrt{n}} \sum_{j=1}^{n}\left\{-\int_{0}^{X_{i}} \frac{d \psi_{\theta}^{-1 \prime}}{d \theta}\{\pi(u)\} \pi(u) d \Lambda^{\sharp}(u)\right\} \frac{\psi_{\theta}^{-1 \prime}\left(S_{1}\left(X_{i}\right)\right)}{\left[\psi_{\theta}^{-1 \prime}\left(S_{1}\left(X_{i}\right)\right)\right]^{3}} \\
\times\left[\int_{0}^{X_{i}}-\psi_{\theta}^{-1 \prime}\{\pi(u)\} d M_{j}(u)+\int_{0}^{X_{i}} \Phi^{\prime}(\pi(u))\left[I\left(X_{j}>u\right)-\pi(u)\right] d \Lambda^{\sharp}(u)\right]+o_{p}(1)
\end{gathered}
$$

and

$$
\begin{aligned}
& \sqrt{n}\left[\hat{S}_{1}\left(X_{i}\right)-S_{1}\left(X_{i}\right)\right] \\
& =\frac{1}{\left[\psi_{\theta}^{-1 \prime}\left(S_{1}\left(X_{i}\right)\right)\right]}\left\{-\int_{0}^{X_{i}} \psi_{\theta}^{-1 \prime}\{\hat{\pi}(u)\} \hat{\pi}(u) \frac{d \bar{N}(u)}{\bar{Y}(u)}\right. \\
& \left.+\int_{0}^{X_{i}} \psi_{\theta}^{-1 \prime}\{\pi(u)\} \pi(u) d \Lambda^{\sharp}(u)\right\} \\
& =\frac{1}{\sqrt{n}} \frac{1}{\left[\psi_{\theta}^{-1 \prime}\left(S_{1}\left(X_{i}\right)\right)\right]}\left[\int_{0}^{X_{i}}-\psi_{\theta}^{-1 \prime}\{\pi(u)\} d \bar{M}(u)\right. \\
& \left.+\frac{1}{\sqrt{n}} \int_{0}^{X_{i}} \sum_{j=1}^{n} \Phi^{\prime}(\pi(u))\left[I\left(X_{j}>u\right)-\pi(u)\right] d \Lambda^{\sharp}(u)\right]+o_{p}(1) \text {. } \\
& =\frac{1}{\sqrt{n}} \frac{1}{\left[\psi_{\theta}^{-1 \prime}\left(S_{1}\left(X_{i}\right)\right)\right]} \sum_{j=1}^{n}\left[\int_{0}^{X_{i}}-\psi_{\theta}^{-1 \prime}\{\pi(u)\} d M_{j}(u)\right. \\
& \left.+\int_{0}^{X_{i}} \Phi^{\prime}(\pi(u))\left[I\left(X_{j}>u\right)-\pi(u)\right] d \Lambda^{\sharp}(u)\right]+o_{p}(1),
\end{aligned}
$$

Hence $\frac{\sqrt{n}}{n}$ Term 1 can be expressed as $\sqrt{n}$ times a linear combination of order $2 \mathrm{U}$ statistics, therefore it is asymptotically normal. Now

$$
\begin{gathered}
\text { Term } 2=-\sum_{i=1}^{n} \hat{S}_{1}\left(X_{i}\right) \exp \left(-\frac{X_{i}}{\hat{\mu}}\right) \frac{X_{i}}{\hat{\mu}^{2}} \frac{d \hat{\mu}}{d \theta}+S_{1}\left(X_{i}\right) \exp \left(-\frac{X_{i}}{\mu}\right) \frac{X_{i}}{\mu^{2}} \frac{d \mu}{d \theta} \\
=-\sum_{i=1}^{n} \exp \left(-\frac{X_{i}}{\mu}\right) \frac{X_{i}}{\mu^{2}} \frac{d \mu}{d \theta}\left[\hat{S}_{1}\left(X_{i}\right)-S_{1}\left(X_{i}\right)\right] \\
+S_{1}\left(X_{i}\right)\left[\exp \left(-\frac{X_{i}}{\hat{\mu}}\right) \frac{X_{i}}{\hat{\mu}^{2}} \frac{d \hat{\mu}}{d \theta}-\exp \left(-\frac{X_{i}}{\mu}\right) \frac{X_{i}}{\mu^{2}} \frac{d \mu}{d \theta}\right]+o_{p}(1 / \sqrt{n})
\end{gathered}
$$




$$
\begin{gathered}
=-\sum_{i=1}^{n} \exp \left(-\frac{X_{i}}{\mu}\right) \frac{X_{i}}{\mu^{2}} \frac{d \mu}{d \theta}\left[\hat{S}_{1}\left(X_{i}\right)-S_{1}\left(X_{i}\right)\right] \\
+S_{1}\left(X_{i}\right) \frac{d \mu}{d \theta}\left[\exp \left(-\frac{X_{i}}{\hat{\mu}}\right) \frac{X_{i}}{\hat{\mu}^{2}}-\exp \left(-\frac{X_{i}}{\mu}\right) \frac{X_{i}}{\mu^{2}}\right] \\
+S_{1}\left(X_{i}\right) \exp \left(-\frac{X_{i}}{\mu}\right) \frac{X_{i}}{\mu^{2}}\left[\frac{d \hat{\mu}}{d \theta}-\frac{d \mu}{d \theta}\right]+o_{p}(1 / \sqrt{n}) . \\
=-\sum_{i=1}^{n} \exp \left(-\frac{X_{i}}{\mu}\right) \frac{X_{i}}{\mu^{2}} \frac{d \mu}{d \theta}\left[\hat{S}_{1}\left(X_{i}\right)-S_{1}\left(X_{i}\right)\right] \\
+S_{1}\left(X_{i}\right) \frac{d \mu}{d \theta}\left[-\frac{X_{i}^{2}}{\mu^{2}} \exp \left(-\frac{X_{i}}{\mu}\right)+\frac{2 X_{i}}{\mu} \exp \left(-\frac{X_{i}}{\mu}\right)\right]\left\{\frac{1}{\hat{\mu}}-\frac{1}{\mu}\right\} \\
+S_{1}\left(X_{i}\right) \exp \left(-\frac{X_{i}}{\mu}\right) \frac{X_{i}}{\mu^{2}}\left[\frac{d \hat{\mu}}{d \theta}-\frac{d \mu}{d \theta}\right]+o_{p}(1 / \sqrt{n}) .
\end{gathered}
$$

Noticing the fact that

$$
\begin{gathered}
\sqrt{n}\left\{\frac{1}{\hat{\mu}}-\frac{1}{\mu}\right\}=\frac{\sqrt{n}}{n} \sum_{j=1}^{n}\left\{\frac{-\log \left(\hat{S}\left(X_{j}\right)\right)}{X_{j}}-E\left(\frac{-\log (S(X))}{X}\right)\right\} \\
=\frac{\sqrt{n}}{n} \sum_{j=1}^{n}\left[\frac{-\log \left(\hat{S}\left(X_{j}\right)\right)}{X_{j}}-\frac{-\log \left(S\left(X_{j}\right)\right)}{X_{j}}\right. \\
\left.+\frac{-\log \left(S\left(X_{j}\right)\right)}{X_{j}}-E\left(\frac{-\log (S(X))}{X}\right)\right] \\
=\frac{\sqrt{n}}{n} \sum_{j=1}^{n}\left[\frac{-\hat{S}\left(X_{j}\right)}{S\left(X_{j}\right) X_{j}}-\frac{-S\left(X_{j}\right)}{S\left(X_{j}\right) X_{j}}\right. \\
\left.+\frac{-\log \left(S\left(X_{j}\right)\right)}{X_{j}}-E\left(\frac{-\log (S(X))}{X}\right)+o_{p}(1)\right] \\
=\frac{1}{n^{3 / 2}} \sum_{j=1}^{n} \sum_{k=1}^{n} \frac{\int_{0}^{X_{j}}-\psi_{\theta}^{-1 \prime}\{\pi(u)\} d M_{k}(u)}{\psi_{\theta}^{-1 \prime}\left(S_{1}\left(X_{j}\right)\right) S_{1}\left(X_{j}\right) X_{j}} \\
+\frac{1}{n^{3 / 2}} \sum_{j=1}^{n} \sum_{k=1}^{n} \frac{\left.\int_{0}^{X_{j}} \Phi^{\prime}(\pi(u))\left[I\left(X_{k}>u\right)-\pi(u)\right] d \Lambda^{\sharp}(u)\right]}{\psi_{\theta}^{-1 \prime}\left(S_{1}\left(X_{j}\right)\right) S\left(X_{j}\right) X_{j}} \\
+\frac{1}{\sqrt{n}} \sum_{j=1}^{n}\left[\frac{-\log \left(S\left(X_{j}\right)\right)}{X_{j}}-E\left(\frac{-\log (S(X))}{X}\right)\right]+o_{p}(1)
\end{gathered}
$$


and

$$
\begin{gathered}
\sqrt{n}\left(\frac{d \hat{\mu}}{d \theta}-\frac{d \mu}{d \theta}\right)=\frac{\sqrt{n}}{\hat{\mu}^{2}} \frac{1}{n} \sum_{j=1}^{n}\left[\frac{-d \hat{S}\left(X_{j}\right) / d \theta}{\hat{S}\left(X_{j}\right) X_{j}}-E\left(-\frac{d S(X) / d \theta}{S(X) X}\right)\right] \\
=\frac{1}{\hat{\mu}^{2}} \frac{\sqrt{n}}{n} \sum_{j=1}^{n}\left[\frac{-d \hat{S}\left(X_{j}\right) / d \theta}{\hat{S}\left(X_{j}\right) X_{j}}+\frac{d \hat{S}\left(X_{j}\right) / d \theta}{S\left(X_{j}\right) X_{j}}-\frac{d \hat{S}\left(X_{j}\right) / d \theta}{S\left(X_{j}\right) X_{j}}\right. \\
\left.+\frac{d S\left(X_{j}\right) / d \theta}{S\left(X_{j}\right) X_{j}}-\frac{d S\left(X_{j}\right) / d \theta}{S\left(X_{j}\right) X_{j}}+E\left(\frac{d S(X) / d \theta}{S(X) X}\right)\right] . \\
=\frac{1}{\hat{\mu}^{2}} \frac{\sqrt{n}}{n} \sum_{j=1}^{n}\left[\frac{-d S\left(X_{j}\right) / d \theta}{X_{j}}\left(\frac{1}{\hat{S}\left(X_{j}\right)}-\frac{1}{S\left(X_{j}\right)}\right)\right. \\
\left.-\frac{1}{S\left(X_{j}\right) X_{j}}\left(d \hat{S}\left(X_{j}\right) / d \theta-d S\left(X_{j}\right) / d \theta\right)-\frac{d S\left(X_{j}\right) / d \theta}{S\left(X_{j}\right) X_{j}}+E\left(\frac{d S(X) / d \theta}{S(X) X}\right)\right] \\
=\frac{1}{\mu^{2}} \frac{\sqrt{n}}{n} \sum_{j=1}^{n}\left[\frac{-\left[d S\left(X_{j}\right) / d \theta\right]^{2}}{S\left(X_{j}\right)^{2} X_{j}}\left(\hat{S}\left(X_{j}\right)-S\left(X_{j}\right)\right)\right. \\
\left.\frac{1}{S\left(X_{j}\right) X_{j}}\left(d \hat{S}\left(X_{j}\right) / d \theta-d S\left(X_{j}\right) / d \theta\right)-\frac{d S\left(X_{j}\right) / d \theta}{S\left(X_{j}\right) X_{j}}+E\left(\frac{d S(X) / d \theta}{S(X) X}\right)\right]+o_{p}(1) .
\end{gathered}
$$

From above expression, we can easily show that $\frac{\sqrt{n}}{n}$ Term 2 can be expressed as $\sqrt{n}$ times a linear combination of order 2 or order $3 \mathrm{U}$ statistics following the same way we have used for proving the asymptotic normality of $\frac{\sqrt{n}}{n}$ Term 1 , hence $\frac{\sqrt{n}}{n}$ Term 2 is asymptotically normal. Similar arguments can be applied to prove the asymptotic normality of $\frac{\sqrt{n}}{n}$ Term 3 and $\frac{\sqrt{n}}{n}$ Term 4 . We can conclude that our parameter estimator is asymptotically normal based on above derivations.

\section{References}

Carrierè, J. (1995) Removing cancer when it is correlated with other causes of death. Biome J. 37, 339-350. 
Clayton, D. G. (1978). A model for association in bivariate life tables and its application in epidemiological studies of familial tendency in chronic disease incidence. Biometrika, 65, 141-51.

Escarela G, Carrierè, J. (2003) Fitting competing risks with an assumed copula. Stat. Methods Med. Res., 12, 333-349.

Fine, J. P., Jiang, H. and Chappell, R. (2001). On semi-competing risks data. Biometrika 88, 907-919.

Fleming, T. R. and Harrington, D. P. (1991) Counting processes and survival analysis. John Wiley \& Sons, Inc.

Genest, C. (1987). Frank's family of bivariate distributions. Biometrika, 74, $549-555$.

Genest, C. and MacKay, R. J. (1986). The joy of copulas: bivariate distributions with uniform marginals. Amer. Statist., 40, 280-283.

Genest, C. and Rivest, L-P. (1993). Statistical inference procedures for bivariate archimedean copulas. J. Amer. Statist. Assoc., 88, 1034-1043.

Heckman, J. J. and Honoré, B. E. (1989). The identifiability of the competing risks model. Biometrika, 76, 325330 .

Hougaard, P. (1986). A class of multivariate failure time distributions. Biometrika, 73, 671-678.

Kaplan, E. L. and Meier, P. (1958) Nonparametric Estimation from Incomplete Observations. J. Amer. Statist. Assoc., 53, 457-481. 
Klein, J and Moeschberger, M (1997). Survival analysis: Techniques for censored and truncated data. Springer (New York).

Lakhal, L., Rivest, L-P., Abdous, B. (2008). Estimating Survival and Association in a Semicompeting Risks Model. Biometrics, 64, 180-188.

Lawless, J. F. and Yilmaz Y.E. (2011) Semiparametric estimation in copula models for bivariate sequential survival times. Biom. J., 53, 779-796.

Nelsen, R. B. (2007). An introduction to copulas. Springer Science \& Business Media.

Oakes, D. (1986). Semiparametric inference in a model for association in bivariate survival data. Biometrika, 73, 353-361.

Rivest, L-P., Wells M.T. (2001). A martingale approach to the copula-graphic estimator for the survival function under dependent censoring. J Multivar Anal, 79, 138-155.

Tsiatis, A. (1975) A nonidentifiability of the problem of competing risks. Proc. Nat. Acad. Sci. USA. $72120-22$.

van der Vaart, A.W. (2007) Asymptotic Statistics. Cambridge University Press.

Wang A. (2012) On the nonidentifiability property of Archimedean copula models under dependent censoring. Stat. and Probabil. Lett. 82, 621-625.

Wang, A (2014). Properties of the Marginal Survival Functions for Dependent Censored Data under an assumed Archimedean Copula. Journal of Multivari- 
ate Analysis, 129, 57-68.

Wang A, Chandra K, Xu R, Sun J (2015) The identifiability of dependent competing risks models induced by bivariate frailty models. Scandinavian Journal of Statististics, 42, 427-437.

Zeng, D., Lin, D. Lin, X. (2008). Semiparametric transformation models with random effects for clustered failure time data. Stat. Sinica 18, 355377.

Zheng, M., Klein, J.P. (1995). Estimates of marginal survival for dependent competing risks based on an assumed copula. Biometrika, 82, 127-138. 